\title{
Recursive Construction of 16-QAM Super-Golay codes for OFDM systems
}

\author{
Masoud Olfat and K. J. Ray Liu \\ Dept. of Electrical and Computer Engineering \\ Univ. of Maryland, College Park, MD 20742
}

\begin{abstract}
In this paper, the Peak to mean Envelope Power (PMEPR) of the OFDM codes generated from an 16-QAM constellation is discussed. Maintaining the same level of error correction properties, these codes achieve higher information rate. A new set of 16-QAM Golay sequences having PMEPR bounded up to $3 \mathrm{~dB}$ is defined. Many recursive structures are found to generate Super Golay sequences. The recursive algorithm is started by a generalized realization of an 16-QAM sequence as a weighted sum of two QPSK sequences. The coding rate and information rate of the generated code is compared to similar works.
\end{abstract}

\section{INTRODUCTION}

Orthogonal Frequency Division Modulation (OFDM) is a parallel data transmission scheme that has recently attracted lots of interest to combat the effect of frequency selective channels in wideband wireless communications.

One of the major hurdles to the widespread use of OFDM is the high Peak to Average Power Ratio(PAPR) of OFDM signals [1]. Usually the transmitters are constrained to a limited peak power. This in turn reduces the average power allowed under OFDM relative to that under single carrier modulation schemes. In addition, to prevent the spectral growth of the OFDM in the form of intermodulation among subcarriers, the transmitter amplifier must operate in a linear region, otherwise, the mobile battery lifetime is reduced.

Many approaches have been proposed to overcome this barrier. The conventional solution to this problem is to use a linear amplifier or to back-off the operating point of nonlinear amplifier, both result in a severe efficiency penalty. Another solution presented in [2] is to simply clip the signal before amplification. This approach causes performance degradation. Another attractive idea was using block coding across the subcarriers and selecting the codewords that minimize or reduce PAPR [3] and [4]. This scheme requires an exhaustive search, and does not address error correction.

In one of the most recent and efficient works done in this area, Golay Complementary Sequences (GCS) [5] are used as codewords to control the modulation of carrier information resulting in OFDM signals with
PAPR at most 2. Davis and Jedwab [6] presented a magnificent work to obtain a large set of length $2^{m}$ binary Golay Complementary Pairs (GCP) from certain second order cosets of the first order Reed-Muller [7] codes . They combined block coding schemes (with all of its properties like efficient encoding and decoding and error correction capabilities) with the use of GCS (with their attractive power control properties). They also went one step further and found $2^{h}$-ary GCP from cosets of an appropriate generalization of the Reed-Muller codes. However, Tarokh and Paterson [8] performed a theoretical analysis for a general coding scheme, having a constellation with equal energy symbols. They found a trade-off between the PAPR, the data rate and the minimum distance of the code. In other words, given the data rate and minimum distance, they found a lower bound for the PAPR that is increased with increasing data rate.

In this paper we will look at the general problem of PAPR reduction in multicarrier systems and specifically try to overcome the limitation on PAPR reduction imposed by coding rate [8]. Here, we relax the assumption of having an equal energy constellation and use QAM for modulation. We define a new version of Golay Complementary Sequences to support these codes. The scheme presented in this paper uses a recursive procedure to build the Super Golay (SGolay) 16-QAM sequences. We generalize the recursive schemes for the case of binary Golay sequences introduced in [5]. Using $M$-QAM modulation allows a better error correcting capabilities over $M$-PSK. Tarokh and Chong in [9] designed a construction method for low PAPR 16-QAM codes that uses the Jedwab's construction for QPSK Golay sequences [6]. The coding rate achieved by this construction is low (about 3\% for 256 subchannels). It should be pointed out that their constructions does not guarantee an upper bound of $3 d B$ for the OFDM PAPR. If they want the PAPR to be bounded up to $3 \mathrm{~dB}$, their coding rate will be cut in half. Tarokh and Rößing, in a related work [10], designed another construction for 16-QAM low PAPR codes. In this work a QAM symbol is represented as a weighted sum of two QPSK symbols. 
The constructed sequences are not Golay, but their PAPR is bounded up to $3.6(=5.6 \mathrm{~dB})$, and the coding rate is twice as the rate for Golay QPSK codes found in [6]. For example for $N=256$ it results in a rate of $9.7 \%$ while the PAPR is guaranteed to be bounded up to 3.6 (5.6dB).

The remainder of this paper is organized as follows. In Section II, we will describe the motivation of the research problem. Section III outlines a proposed structure to achieve low PAPR for a general constellation. In Section IV a seed for our recursive procedure is introduced. In Section $\mathrm{V}$ we present some simulation results and compare our construction with some existing works and finally Section VI concludes the paper.

\section{EQUAL-ENERGY GOLAY CODES}

Channel coding is a mean to perform the PAPR reduction and some error correction for OFDM systems simultaneously. If $x$ is an $N$-valued complex sequence applied to IFFT block in an OFDM transmitter with frequency separation $\Delta f$, the instantaneous envelope power of the OFDM signal is

$$
p_{x}(t)=\sum_{i=0}^{N-1} \sum_{u=0}^{N-1} x_{i} x_{u}^{*} \exp [j 2 \pi(i-u) \Delta f t] .
$$

Definition 1: The Cross-Correlation of two $N$ valued complex sequences $x$ and $y$ with replacement $0 \leq l \leq N-1$ is defined as

$$
C_{l}(x, y)=\sum_{i=0}^{N-l-1} x_{i}^{*} y_{i+l}
$$

and $C_{-l}(x, y)=\left(C_{l}(y, x)\right)^{*}$.

Definition 2: The Auto-Correlation of an Nvalued complex codeword $x$ with replacement $1-N \leq l \leq N-1$ is $A_{l}(x)=C_{l}(x, x)$ or

$$
A_{l}(x)=\sum_{i=0}^{N-l-1} x_{i}^{*} x_{i+l} .
$$

Using these definitions, the instantaneous envelope power of the OFDM signal can be restated as

$$
p_{x}(t)=A_{0}(x)+\sum_{|l|=1}^{N-1} A_{l}(x) e^{j 2 \pi l \Delta f t} .
$$

The average power of the OFDM signal is $A_{0}(x)$, which is the same as the power of the codeword $x$ (by Parseval equation) and is denoted by $P_{x}$. The maximum possible value for PAPR in an OFDM system is equal to the number of subchannels, $N$.

Definition 3: Two $N$-valued complex sequences $x$ and $y$ are called Golay Complementary Pairs $(G C P)$ if

$$
A_{l}(x)+A_{l}(y)=0 \quad \forall l \neq 0 .
$$

We show this by $x \sim y$. Each of the sequences $x$ and $y$ is called a Golay Complementary Sequence (GCS).
Assume that the sequences $x$ and $y$ are GCP and have the same power $\left(A_{0}(x)=A_{0}(y)\right)$. The instantaneous envelope power is non-negative at all times, therefore

$$
P A P R(x) \triangleq \frac{\max _{t}\left\{p_{x}(t)\right\}}{P_{x}} \leqslant 2=3 d B .
$$

Consequently, if we choose the codewords from a set of Golay sequences, the PAPR is bounded up to $3 \mathrm{~dB}$.

Drawing the elements of the codeword from an equalenergy constellations, Davis and Jedwab [6] proved that each of the $\frac{m !}{2}$ cosets of the first order Reed-Muller code [7], $R M_{2^{h}}(1, m)$, having a coset representation of the form $2^{h-1} \sum_{k=0}^{m-1} x_{\pi(k)} x_{\pi(k+1)}$ comprises $2^{h(m+1)}$ Golay sequences over $\mathcal{Z}_{2^{h}}$ of length $2^{m}$, where $\pi$ is a permutation of the symbols $\{1,2, \ldots, m\}$ and $h>1$. This generates $\frac{m !}{2} 2^{h(m+1)}$ Golay sequences of length $2^{m}$. Encoding this code can be done by a very simple digital counter, and its decoding can be done very efficiently by using Sylvester-Hadamard transformation [7].

They also introduced a construction for building at least $2^{h(m+2)} \frac{m !}{2}$ Golay complementary pairs over $\mathcal{Z}_{2^{h}}$ of length $2^{m}$.

\section{PAPR REDUCTION FOR THE NON-EQUAL ENERGY CONSTELLATION}

Given the length of each codeword, minimum Euclidian distance, and maximum PAPR, Tarokh and Paterson [8] found a lower bound for achievable coding rate. On the other hand, given the length of each codeword, coding rate and minimum Euclidian distance, they found a lower bound for the PAPR. The lower bound for the PAPR increases by increasing the coding rate.

The need for low PAPR and at the same time overcoming the lower bound of rate for equal-power codes has motivated us to investigate the non-equal power codes that achieve low PAPR. To do this we define a special case of GCSs.

Definition 4: Two $N$-valued complex sequences $x$ and $y$ are called Super Golay Complementary Pairs $(S G C P)$ if they are Golay pairs and $P_{x}+P_{y} \leq 2 N P_{a v}$, when $P_{a v}$ is the average power of the constellation. We show this by $x \approx y$. Each of the sequences $x$ and $y$ is called Super Golay Complementary Sequence (SGCS)

For a special case, It has been proved in [10] that if the 16-QAM sequences are realized as a sum of two sequences chosen from an equi-probable set of QPSK codewords, then the mean envelope power of the transmitted OFDM symbol is $P_{a v}$, the average power of the 16-QAM constellation. This fact can be easily generalized to our structures introduced in Section IV. Therefore, by virtue of (2) and the fact that the instantaneous power of each codeword is always non-negative we can see that the PAPR achieved by any SGCS is bounded up by $3 d B$. 
Next, we would like to find a construction method for SGolay codes. If $x$ and $y$ are two $N$-valued sequences, we show the inverse of $x$ by $\hat{x}$, the elementwise conjugate of $x$ by $x^{*}$, the concatenation of $x$ and $y$ by $x \mid y$, and the interleaving of $x$ and $y$ by $x \downarrow y$. Also we show the sequence $\left(x_{1},-x_{2}, x_{3}, \ldots,(-1)^{N-1} x_{N}\right)$ by $x^{\prime}$. The following theorem can be seen very easily from Definition 2.

Theorem 5: The property of being Super Golay complementary pairs is invariant under the following transformations:

- Reflection w.r.t the origin.

- Reflection w.r.t both axes.

- Reflection w.r.t the bisectors of all regions.

- Rotation of one or both sequences.

Theorem 6: If $x \approx y$ then
a. $x^{\prime} \approx y^{\prime}$.
b. $\hat{x} \approx \hat{y}$
c. $x \approx \hat{y}^{*}$

Proof: If $x \approx y$ then

a . The $k$ th member of the sequence $x^{\prime}$ is $x_{k}^{\prime}=(-1)^{k} x_{k}$, therefore using Definition 2.

$$
A_{l}\left(x^{\prime}\right)=(-1)^{l} A_{l}(x) .
$$

$\mathrm{b}$. The $k$ th member of the sequence $\hat{x}$ is $\hat{x}_{k}=x_{N-1-k}$, therefore using Definition 2.

$$
\begin{aligned}
A_{l}(\hat{x}) & =\sum_{i=0}^{N-l-1} \hat{x}_{i+l} \hat{x}_{i}^{*}=\sum_{i=0}^{N-l-1} x_{N-l-i-1} x_{N-i-1}^{*} \\
& =\sum_{k=0}^{N-l-1} x_{k+l}^{*} x_{k}=\left(A_{l}(x)\right)^{*}
\end{aligned}
$$

c Using $A_{l}(-x)=A_{l}(x)$ and (5), the statement is concluded.

Theorem 7: If $x \approx y$ then
a. $x|y \approx x|-y$
b. $x \downarrow y \approx x \downarrow-y$

Proof: The items are proved separately:

a . It is easy to see that

$$
A_{l}(x \mid y)=A_{l}(x)+A_{l}(y)+\sum_{i=0}^{l-1} x_{N-1-i}^{*} y_{l-1-i}
$$

and therefore

$$
A_{l}(x \mid y)+A_{l}(x \mid-y)=2\left(A_{l}(x)+A_{l}(y)\right)=0 .
$$

b. if $l=2 k$ then

$$
A_{l}(x \downarrow y)=A_{l / 2}(x)+A_{l / 2}(y)=0 .
$$

If $x \approx y$, then $x \approx-y$, and since $A_{l}(-x)=$ $A_{l}(x), \quad$ then $\quad A_{l}(x \downarrow-y)=0 . \quad$ Therefore,

$$
\begin{aligned}
& A_{l}(x \downarrow y)+A_{l}(x \downarrow-y)=0 . \\
& \text { If } l=2 k+1, \text { then } \\
& \qquad A_{l}(x \downarrow y)=\sum_{i=0}^{N-1-\frac{l-1}{2}} x_{i}^{*} y_{i+\frac{l-1}{2}} .
\end{aligned}
$$

and therefore $A_{l}(x \downarrow y)+A_{l}(x \downarrow-y)=0$.

By applying the transformations defined in Theorems 5 and 6 to the statements of Theorem 7, we can build a set of structures that create $2 N$-valued Super Golay pairs from $N$-valued ones. Specifically, if $x \approx y$, each with size $N$, then the following sequences are Super Golay pairs $([j]$ means multiplying the sequence by $j$ is optional) :

$$
\begin{aligned}
& \text { 1) } \pm[j](x \mid y) \quad \approx \pm[j](x \mid-y) \\
& \text { 2) } \pm[j](x \downarrow y) \quad \approx \pm[j](x \downarrow-y) \\
& 3) \pm[j](x \mid y) \quad \approx \pm[j]\left(\hat{y}^{*} \mid-\hat{x}^{*}\right) \\
& \text { 4) } \pm[j](x \downarrow y) \quad \approx \pm[j]\left(\hat{y}^{*} \downarrow-\hat{x}^{*}\right) \\
& 5) \pm[j](x \downarrow-y) \approx \pm[j]\left(\hat{y}^{*} \downarrow \hat{x}^{*}\right)
\end{aligned}
$$

However, because of the special structure of 16-QAM constellation, many of these constructions yield similar sequences. For example reversing the role of $x$ and $y$ will not yield new pairs. If the number of $N$-valued pairs is $M$, the first structure yields $4 M$ of $2 N$-valued SGolay pairs and this is true for the second structure too. We have performed a simulation for the pairs with size 8 and obtained the same result. In general each pair with size $N$ yields 32 pairs each with size $2 N$. This is similar to the Reed-Muller codes used for equal-power Golay sequences. Reed-Muller codes of degree $r+1$ and length $2^{m+1}$ can be constructed from two $2^{m}$-length Reed-Muller codes, one in degree $r$ and one in degree $r+1$.

\section{SGOLAY 16QAM PAIRS FROM QPSK PAIRS}

In this section we will look at an important question which is how to start the recursive construction. To this end, we will use the relation that Tarokh and Rößing [10] used. They represented a 16-QAM symbol by a weighted sum of two QPSK symbols. We will generalize their observation and find a construction that builds 16QAM SGolay sequences from QPSK Golay sequences. Let's define QPSK symbols as the set

$$
Q P S K=\left\{\exp \left[j\left(\frac{k \pi}{2}+\frac{\pi}{4}\right)\right], \mid k \in \mathcal{Z}_{2^{h}}\right\}
$$

Using Definitions 1 and 2, the following lemma can be proved easily,

Lemma 8: For any two sequences $x$ and $y$ and any two complex numbers $\alpha$ and $\beta$

$$
\begin{aligned}
A_{l}(\alpha x+\beta y) & =|\alpha|^{2} A_{l}(x)+|\beta|^{2} A_{l}(y) \\
& +\alpha \beta^{*} C_{l}(x, y)+\alpha^{*} \beta C_{l}(y, x) .
\end{aligned}
$$


Theorem 9: If $x$ and $y$ are $N$-valued QPSK Golay pairs, and $|\alpha|=|\beta|$, then each of the following pairs are 16-QAM Super Golay sequences:

$$
\begin{aligned}
& 1 . c=\alpha(x+2 y) \quad \text { and } t=\beta(-2 x+y) \\
& 2 . c=\alpha(x-2 y) \text { and } t=\beta(2 x+y) \\
& 3 . c=\alpha(x+2 j y) \text { and } t=\beta(2 j x+y) \\
& 4 . \quad c=\alpha(x-2 j y) \text { and } t=\beta(-2 j x+y)
\end{aligned}
$$

Proof: We will prove the result for the third item. The rest can be proved similarly. Using Theorem 8 , for each nonzero $l$,

$$
A_{l}(c)+A_{l}(t)=5|\alpha|^{2}\left(A_{l}(x)+A_{l}(y)\right)=0
$$

Therefore $c$ and $t$ are Golay pairs. It is easy to see that each of these sequences are actually a 16-QAM sequence, when the average power of the constellation is $P_{a v}=5|\alpha|^{2}$. If we denote the Hermition of $x$ by $x^{H}$, and consider the fact that the power of both $x$ and $y$ is $N$, then

$$
\begin{aligned}
P_{c}+P_{t} & =|\alpha|^{2}\left(\|x+2 j y\|^{2}+\|2 j x+y\|^{2}\right) \\
& =5|\alpha|^{2}\left(\|x\|^{2}+\|y\|^{2}\right)=10 N|\alpha|^{2}=2 N P_{a v} .
\end{aligned}
$$

Therefore, by Definition 4, $c$ and $t$ are Super Golay sequences.

Theorem 9 suggests a starting point for the proposed recursive construction. If we limit ourselves to an 16-QAM construction with $P_{a v}=10$, then $\alpha$ and $\beta$ can be chosen from the set $\{\sqrt{2},-\sqrt{2}, j \sqrt{2},-j \sqrt{2}\}$, and therefore for each of the $2^{h(m+2)} \frac{m !}{2}$ Golay complementary pairs over $\mathcal{Z}_{2^{h}}$ of length $2^{m}$, there are 64 Super Golay 16-QAM pairs. However, some of these pairs are repeated. As an example if $(c \sim t)$, then $(-c \sim-t)$ and therefore we do not need to multiply the two sequences in the first construction of Theorem 9 by $-\sqrt{2}$. Eliminating these repeated sequences, the number of 16-QAM Super Golay pairs generated from each QPSK Golay pair is 16. Therefore we can build $2^{4+h(m+2)} \frac{m !}{2}$ distinct QAM Super Golay pairs over $\mathcal{Z}_{2^{h}}$ of length $2^{m}$. For $m=2$ and QPSK symbols $(h=2)$, this translates to 4096 pairs. Through exhaustive search, we have found that there are exactly 12032 Super Golay pairs and Theorem 9 builds 4096 of them.

We were able to come up with some structures that build in average 32 new $2 N$-valued SGolay pairs from one $N$-valued SGolay pair. Therefore, starting from 4valued codes, our construction is able to achieve at least

$$
R=\frac{12+5 \log _{2} N / 4}{4 N} .
$$

code rate for $N$ OFDM subchannels.

For 128 subchannels, this achieves $7.3 \%$ code rate. Although not still acceptable, this is about $11 \%$ improvement over Tarokh-Chong's work [9].
The achievable code rate is about $20 \%$ below the equal power Golay codes constructed by Jedwab and Davis [6]. However, because of using 16-QAM constellation, the information rate achieved by these structures is twice as the information rate achieved by Jedwab's construction, while the error correction properties of the code is maintained.

The structure we proposed is for general Super Golay codes, regardless of the constellation. However, we have focused on 16-QAM constellation for the sake of simulation. This scheme can be generalized to higher order QAM constellations, like 64-QAM which is used in IEEE WLAN standards like IEEE802.11a .

To find a construction method for low PAPR 64-QAM sequences we can use the concept of Golay sets. They are defined as a set of sequences whose autocorrelations with any nonzero replacement add up to zero and therefore their PAPR is bounded up by the size of the set, if they are taken from an equal energy constellation. Paterson has shown that [11] the $2^{k+1}$-size equal power Golay set can be represented by some certain cosets of $R M_{2^{h}}(1, m)$ in $R M_{2^{h}}(2, m)$.

The following lemma which can be proved easily using the Definitions 1 and 2 is a generalization of Lemma 8.

Lemma 10: For any $n$ sequences $x^{i}$ and any $n$ complex numbers $\alpha^{i}, i=1, \ldots n$

$A_{l}\left(\sum_{i=1}^{n} \alpha_{i} x^{i}\right)=\sum_{i=1}^{n}\left|\alpha_{i}\right|^{2} A_{l}\left(x^{i}\right)+\sum_{\substack{i=1 \\ i \neq j}}^{n} \sum_{\substack{j=1 \\ j}}^{n} \alpha_{i} \alpha_{j}^{*} C_{l}\left(x^{i}, x^{j}\right)$.

Using this Lemma, the following theorem can be used to generate 64-QAM Super Golay sets (The sum of the powers is $\left.n N P_{a v}\right)$.

By generalizing Lemma 8, the following theorem can be proved to generate 64-QAM Super Golay sets:

Theorem 11: If $\left\{x^{i} \mid i=1 \ldots 4\right\}$ comprises a $N$ valued QPSK Golay set, and $\left|\alpha_{i}\right|$ is constant for all $i=1, \ldots 4$, then the following is a 64-QAM Super Golay set and therefore, the PAPR of each element is at most $4(6 d B)$ :

$$
\left\{\alpha_{1}\left(4 x^{1}+j x^{2}-j x^{3}-x^{4}\right), \alpha_{2}\left(4 x^{2}+j x^{1}-x^{3}-j x^{4}\right),\right.
$$$$
\left.\alpha_{3}\left(4 x^{3}-j x^{1}+x^{2}-j x^{4}\right), \alpha_{4}\left(4 x^{4}+x^{1}-j x^{2}-j x^{3}\right)\right\} .
$$

Note that, for the super Golay sets, the sum of the powers is not $2 N P_{a v}$ anymore, but it is $n N P_{a v}$, when $n$ is the number of sequences comprising a Golay set.

\section{Simulation}

We have performed a search to find all of QPSK Golay pairs and 16-QAM SGolay pairs of size 4. The total number of QPSK Golay pairs is 256 which is exactly compliant by the formula given in [6] $\left(2^{2(m+2)} \frac{m !}{2}\right.$ distinct QPSK Golay pairs with size 4). Using the representations in Theorem 9, We have been able to build 4096 


\begin{tabular}{|c|c|c|}
\hline Scheme & size 4 SGPS & size 8 SGPs built by Theorem 7 \\
\hline 1 & 12032 & 385020 \\
\hline 2 & 4096 & 131720 \\
\hline
\end{tabular}

TABLE I

NUMBER OF CONSTRUCTED 8-VALUED SGOLAY PAIRS.

\begin{tabular}{|c|c|c|c|}
\hline Scheme & Max PMEPR & Code rate & Information rate \\
\hline Recursive1 & $3 \mathrm{~dB}$ & 0.3438 & 1.375 \\
\hline Recursive2 & $3 \mathrm{~dB}$ & 0.375 & 1.5 \\
\hline Jedwab1 & $3 \mathrm{~dB}$ & 0.31 & 0.62 \\
\hline Jedwab2 & $6 \mathrm{~dB}$ & 0.47 & 0.94 \\
\hline Paterson & $6 \mathrm{~dB}$ & 0.563 & 1.126 \\
\hline Chong1 & $3 \mathrm{~dB}$ & 0.2954 & 1.1817 \\
\hline Chong2 & $5.56 \mathrm{~dB}$ & 0.3053 & 1.2212 \\
\hline
\end{tabular}

TABLE II

RATES AND PMEPRS FOR SIZE 16.

SGolay pairs with size 4 . The total number of 4 -valued SGolay pairs is 12032 . Table I compares the number of 8 -valued SGolay pairs obtained from these two sets. In both cases, each Golay pair with size 4 yields 32 new SGolay pairs with size 8 . In this table, scheme 1 means the total number of SGolay pairs and scheme 2 means the number of SGolay pairs built by Theorem 9. Table II and III compare the code rate, information rate and the achievable PMEPR of the proposed recursive structure with the same values reported in [6], [11], and [9] using QPSK constellation for 16 and 32 subcarriers. In the rows represented by "Jedwab1" the second order ReedMuller cosets of the form $2^{h-1} \sum_{k=0}^{m-1} x_{\pi(k)} x_{\pi(k+1)}$ are used and therefore the constructed codewords are Golay and their PMEPR is bounded up by $3 \mathrm{~dB}$. However, in the scheme represented by "Jedwab2" other forms of second order Reed-Muller cosets are also allowed. This causes the PMEPR of the codeword to exceed 3dB. At the row represented by "Paterson", the concept of Golay sets are used. It is proved in [11] that a Golay set of size $2^{k+1}$ achieve the maximum PMEPR of $2^{k+1}$. The rows represented by "Recursive1" is using the 4-valued 16QAM SGolay pairs generated from QPSK pairs as the seed, while "Recursive2" scheme uses the total 4-valued 16-QAM codes generated by exhaustive search, as the seed.

\section{CONCLUSION AND FUTURE WORK}

In this paper we focused on the construction of a recursive scheme that allows us to build all of the Super Golay sequences with a specific size. We formed some structures to obtain this code from Super Golay sequences with half size. One of the future directions of this work is to search for more structures. The more structure we obtain, the bigger number of SGolay codes we can cover and the higher coding rate we can achieve.

\begin{tabular}{|c|c|c|c|}
\hline Scheme & $P M E P R$ & Code rate & Information rate \\
\hline Recursive1 & $3 \mathrm{~dB}$ & 0.2109 & 0.84375 \\
\hline Recursive2 & $3 \mathrm{~dB}$ & 0.2266 & 0.9063 \\
\hline Jedwab1 & $3 \mathrm{~dB}$ & 0.19 & 0.38 \\
\hline Jedwab2 & $6 \mathrm{~dB}$ & 0.31 & 0.62 \\
\hline Paterson & $6 \mathrm{~dB}$ & 0.375 & 0.75 \\
\hline Chong1 & $3 \mathrm{~dB}$ & 0.1835 & 0.7341 \\
\hline Chong2 & $5.56 \mathrm{~dB}$ & 0.3053 & 1.2212 \\
\hline
\end{tabular}

TABLE III

RATES AND PMEPRS FOR SIZE 32

The construction started from QPSK Golay sequences which are efficiently created using $2^{\text {nd }}$ order cosets of $R M_{2^{h}}(1, m)$. Although the information rate is higher than the existing works in this context, it is not still an acceptable rate. Another future direction of this work is to find the possible trade-off between the coding rate and PAPR.

The structure we propose is for general Super Golay codes, regardless of the constellation. We also presented a scheme that generates 64-QAM sequences with PAPR bounded up by 4 .

A natural future direction of this work we aim to do is to find an encoding and decoding method and also analyze the Euclidian distance structure of the construction.

\section{REFERENCES}

[1] R. van Nee, "OFDM codes for peak-to average power reduction and error correction," Proc. of IEEE Globecom'96, pp. 740-744, 1996.

[2] X. Li and L. J. Cimini, "Effects of clipping and filtering on the performance of OFDM," Proc. of IEEE Vehicular. Tech. Conf., pp. 1634-1638, May 1997.

[3] A. E. Jones, T. A. Wilkinson, and S. K. Barton, "Block coding scheme for reduction of peak to mean envelope power ratio of multicarrier transmission scheme," Electronic Lett., vol. 47, no. 25, pp. 2098-2099, Dec. 1994.

[4] A. E. Jones and T. A. Wilkinson, "Combined coding for error control and increased robustness to system nonlinearties in OFDM," IEEE 46th Vehicular technology Conf., Atlanta, GA, pp. 904-908, Apr/May. 1996.

[5] M. J. E. Golay, "Complementary series," IRE Trans. On Information Theory, pp. 82-87, Apr. 1961.

[6] J. A. Davis and J. Jedwab, "Peak-to-mean power control in OFDM, Golay complementary sequences, and Reed-Muller codes." IEEE Trans. On Information Theory, vol. 45, pp. 23972417, Nov. 1999.

[7] S. B. Wicker, Error control systems for digital communication and storage. Prentice Hall, New Jersey, 1995.

[8] K. G. Paterson and V. Tarokh, "On the existence and construction of good codes with low peak-to-average power ratios," IEEE Trans. on Information Theory, vol. 46, pp. 1974-1987, 2000.

[9] C. V. Chong and V. Tarokh, "Two constructions of 16-QAM Golay Complementary Sequences," http://www.mit.edu/ vahid.

[10] C. Rößing and V. Tarokh, "A construction of OFDM 16-QAM sequences having low peak powers," IEEE Trans. On Information Theory, vol. 47, no. 5, pp. 2091-2093, Jul. 2001.

[11] K. G. Paterson, "Generalized Reed-Muller codes and power control in OFDM modulation," IEEE Trans. On Comm., vol. 46, no. 1, pp. 104-120, Jan. 2000. 